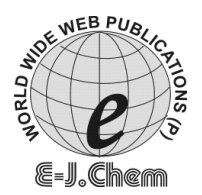

http://www.e-journals.net
ISSN: 0973-4945; CODEN ECJHAO

E-Journal of Chemistry

Vol. 5, No.4, pp. 914-917, October 2008

\title{
Chemoselective Hydrogenation of Aromatic Nitro Compounds Using Diammonium Hydrogen Phosphite and Commercial Zinc Dust
}

\author{
K. ANIL KUMAR, K. S. SHRUTHI, \\ NAGARAJA NAIK and D. CHANNE GOWDA* \\ Department of Studies in Chemistry, \\ University of Mysore, Manasagangothri, Mysore, 570006, India. \\ dcgowda@yahoo.com; Fax: 0921-0821-2419363
}

Received 24 March 2008; Accepted 5 June 2008

\begin{abstract}
The aromatic nitro compounds are reduced to their corresponding amines at room temperature in good yields by employing diammonium hydrogen phosphite as hydrogen donor and zinc as catalyst. The hydrogenation is fast and selective in the presence of the other sensitive functionalities such as halogens, $-\mathrm{OH},-\mathrm{NH}_{2},-\mathrm{OCH}_{3},-\mathrm{CN},-\mathrm{COCH}_{3},-\mathrm{COOH},-\mathrm{COOR}$ etc. It was observed that, this system is equally competitive with existing methods.
\end{abstract}

Keywords: Commercial zinc dust, Diammonium hydrogen phosphite, Aromatic nito compounds, Catalytic transfer hydrogenation.

\section{Introduction}

Aromatic amines are an important class of compounds frequently used as key intermediates in the synthesis of pharmaceutical products, dyestuff and polymers ${ }^{1}$. Various methods have been developed for the preparation of aromatic amines from the corresponding aromatic nitro compounds $\mathrm{s}^{2-4}$. The methods employed generally are metal/acid reduction ${ }^{5}$, catalytic hydrogenation ${ }^{6}$, electrolytic reduction ${ }^{7}$, homogeneous catalytic transfer hydrogenation ${ }^{8}$, heterogeneous catalytic transfer hydrogenation ${ }^{9}$ etc., are in practice. However, these methods have one or more limitations. In recent years, metal mediated reactions have wide scope in organic synthesis because of their simple work-up and selectivity. Several methods have been developed for the reduction of nitro compounds based on the use of metals from our laboratory as well as from other laboratories ${ }^{10-20}$. Diammonium hydrogen phosphite was introduced by Rose $e t a l .^{21}$ as a reducing agent in a number of reactions and also as a corrosion inhibitor for lubricating grease. 
In this context, we wish to report a rapid, selective and simple reduction of substituted nitro compounds to corresponding amino derivatives by using low cost commercial zinc dust and diammonium hydrogen phosphite ${ }^{21}$ at room temperature in methanol medium (Scheme 1). This new system reduced with ease a wide variety of nitro compounds to corresponding amines. Many primary and secondary functional groups like halogens, carboxylic acid, phenol, ester, nitrile etc., are tolerated.

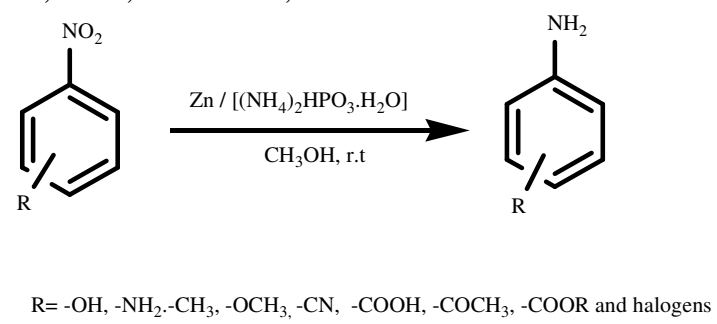

Scheme 1

\section{Experimental}

Materials

All the nitro compounds were purchased from Aldrich Chemical Company (USA) and zinc dust from SISCO Research Laboratories Pvt. Ltd., Bombay (India). Diammonium hydrogen phosphite was prepared according to the published procedure ${ }^{21}$.

All the solvents were of analytical grade or were purified according to standard procedures. TLC was performed on silica gel plates obtained from Whatman Inc with the eluting systems chloroform:methanol(90:10) and chloroform:methanol(95:05). For preparative TLC, the plates were prepared from Kieselgel 60GF254 Merck, Darmstadt, and for column chromatography 60-120 mesh silica gel was used. The melting points were determined by using a Thomas-Hoover melting point apparatus and are uncorrected. IR spectra were recorded on SHIMADZU-FTIR-8300 spectrometer.

\section{General procedure for the preparation of aromatic amines}

A mixture of aromatic nitro compound $(5 \mathrm{mmol})$ and commercial $\mathrm{Zn}$ dust $(6 \mathrm{mmol})$ in methanol $(5 \mathrm{~mL})$ was stirred with diammonium hydrogen phosphite $(10 \mathrm{mmol})$ at room temperature. The reaction was exothermic. After completion of the reaction monitered by (T.L.C), the mixture was filtered off. The organic layer was evaporated and residue was dissolved in $\mathrm{CHCl}_{3}$ or ether $(2 \times 30 \mathrm{~mL})$. The extract was washed with saturated $50 \%$ sodium chloride solution $(30 \mathrm{~mL})$. The organic layer was dried over anhydrous $\mathrm{Na}_{2} \mathrm{SO}_{4}$ and the solvent was evaporated under reduced pressure to obtain the desired amino compound.

\section{Results and Discussion}

The result of this reduction of various nitroarenes was shown in Table 1. In most cases the reaction was over within 5-20 min. The usual side products of nitro reduction such as azoxy, azo, and hydrazo compounds were not observed in the final product. At the same time, it was also noteworthly that the present method was highly chemoselective and some sensitive functional groups such as $-\mathrm{Cl}$, $-\mathrm{Br}$ and $-\mathrm{COOC}_{2} \mathrm{H}_{5}$ did not undergo any change under the reaction conditions. Moreover, many other substituted groups, such as $-\mathrm{CH}_{3},-\mathrm{OH},-\mathrm{OCH}_{3}$, were intact during the reaction.

In order to test the selectivity, reduction was attempted with 4-nitroacetophenone, 4-nitrophenylacetonitrile and 4-nitrobenzoic acid gave the corresponding anilines without 
affecting the other reducible groups. Even the reduction of chloronitrobenzene showed high selectivity for chloroanilines without any dehalogenation of chloronitrobenzene. It is worth to note that; all the substituted nitro compounds reduced by this system were obtained in good yields.

Table1. Reduction of nitroarenes to anilines with diammonium hydrogen phosphite and commercial zinc dust in methanol.

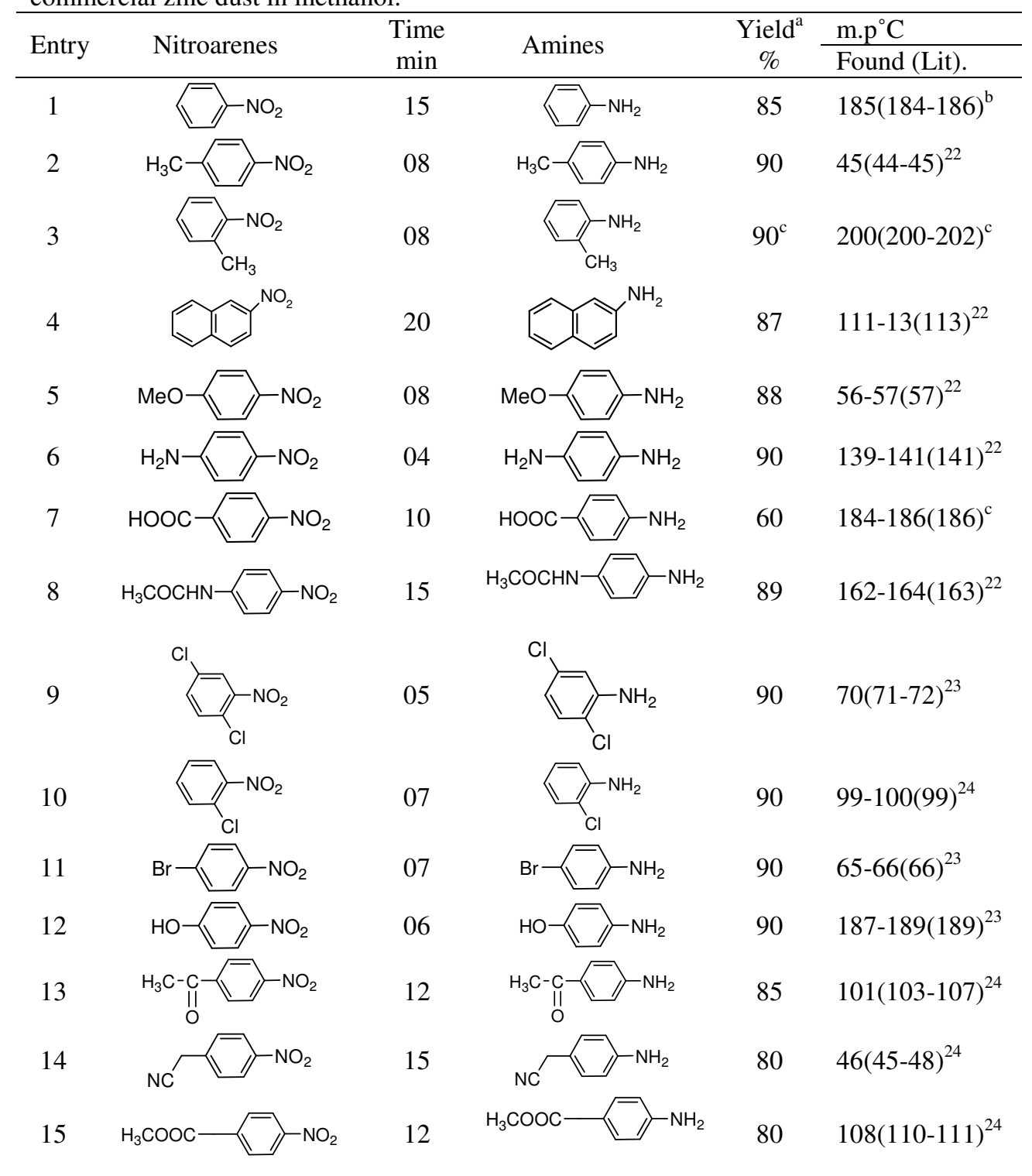

${ }^{a}$ Isolated yields are based on single experiment and the yields were not optimized;

${ }^{b}$ Boiling point; ${ }^{c}$ Isolated as benzoyl derivative.

And also a controlled experiment was carried using substituted nitro compounds with diammonium hydrogen phosphite but without zinc dust, did not yield the desired product. Furthermore, an attempted reduction of a substituted nitro compounds using zinc dust in the 
absence of diammonium hydrogen phosphite, did not yield the desired product, even if the reaction mixture was stirred for more than 24 hours. This clearly confirms that methanol severs as solvent and not as hydrogen source.

\section{Conclusion}

In conclusion, we report here a novel approach for the preparation of aromatic amines from the corresponding aromatic nitro compounds using diammonium hydrogen phosphite in the presence of zinc dust. This method is mild, exceedingly efficient and highly selective. The obvious advantages of proposed method over earlier methods are: (i) selective reduction of nitro compounds, in the presence of other reducible or hydrogenolysable groups, (ii) ready availability and ease of operation, (iii) rapid reduction, (iv) high yields of substituted amines, (v) avoidance of strong acid media, (vi) no equipment of pressure apparatus, (vii) cost effectivity and (viii) prevention of unwanted by products such as, hydroxylamines, nitroso, hydrazo, and azo compounds.

The catalyst is non-pyrophoric in nature and other interesting behaviors of zinc dust lies in fact that it can be recycled after simple washing with ether and dilute $\mathrm{HCl}$, rendering thus process is more economic. The present method offers an economical, safe, and environmentally benign alternative to available procedures.

\section{References}

1. Ram S and Ehrenkaufer R E, Tetrahedron Lett., 1984, 25, 3415.

2. Yuste F, Saldana and Walls F, Tetrahedron Lett., 1982, 23, 147.

3. Lyle R E and La Mattina J L, Synthesis, 1974, 726.

4. Ho T L and Wang C M, Synthesis, 1974, 45.

5. (a) House H O, Modern Synthesis Reactions, $2^{\text {nd }}$ Ed.; Benzamin Inc, U. S. A., 1972, 145; (b) Merlic C A, Motamed and Quinn B, J Org Chem., 1995, 60, 3365.

6. (a) Rylander P N, Hydrogenation Methods, Academic Press, New York, 1985, 365.

(b) Tafesh A M and Weigunty, Chem Rev., 1996, 96, 2035.

7. Popp F D and Schultz H P, Chem Rev., 1962, 62, 19.

8. Harmon R E, Gupta S K and Brown D J, Chem Rev., 972, 73, 21.

9. Johnstone R A W, Willy A H and Entwistle I D, Chem Rev., 1985, 85, 129.

10. Yoo B W, Choi J W, Hwang S K, Kim D Y, Back H S, Choi K I and Kim J H, Synth Commun., 2003, 33, 2985.

11. Wang L, Pin-Hua L and Zhao-Qin J, Chinese J Chem., 2003, 21, 222.

12. Bhaumik K and Akamanchi K G, Can J Chem., 2003, 81, 197.

13. Ragaini F, Cenini S and Gasperini M, J Mol Cat., 2001, 74, 51.

14. Desai D G, Swami S S, Dabhade S K and Ghagare M G, Synth Commun., 2001, 31, 1249.

15. Basu M K, Becker F F and Banik B K, Tetrahedron Lett., 2000, 41, 5603.

16. Wang L, Pinhua L, Zongtao W, Yan J, Min W and Ding Y, Synthesis, 2003, 13, 2001.

17. Khan F A, Dash J, Sudheer C and Gupta R K, Tetrahedron Lett., 2003, 44, 7783.

18. Gowda D C, Mahesha B and Gowda S, Indian J Chem., 2001, 40B, 75-77.

19. Gowda D C, Srinivasa G R and Abiraj K, Indian J Chem., 2003, 42B, 2882-2884.

20. Gowda D C and Gowda S, Indian J Chem., 2000, 39B, 709-711.

21. (a) Gmelin handbook of inorganic and organometallic chemistry, Ammonia 8th Ed.; 1936, 416-417; (b) De Fourcroy A F, Vauquelin L M and Rose H, Journ Polyt., 1795, 4, 655.

22. Vogel A I, Text Book of Practical Organic Chemistry, 5th Ed.; Addison Wesley Longman Limited, UK, 1997.

23. The Merck Index, $11^{\text {th }}$ Ed.; Merck \& Co., Inc., USA, 1989.

24. Hand Book of Fine Chemicals Aldrich 2007-08 


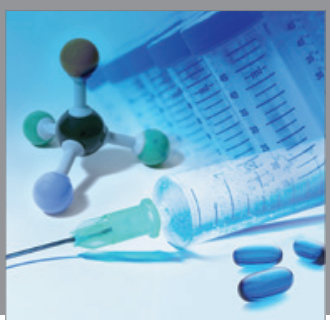

International Journal of

Medicinal Chemistry

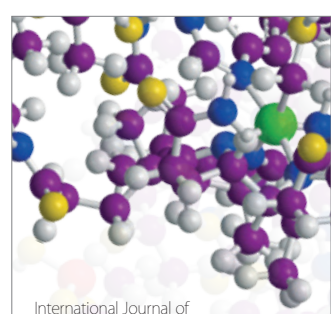

Carbohydrate Chemistry

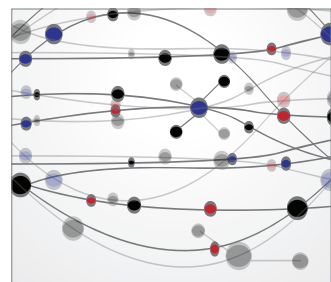

The Scientific World Journal
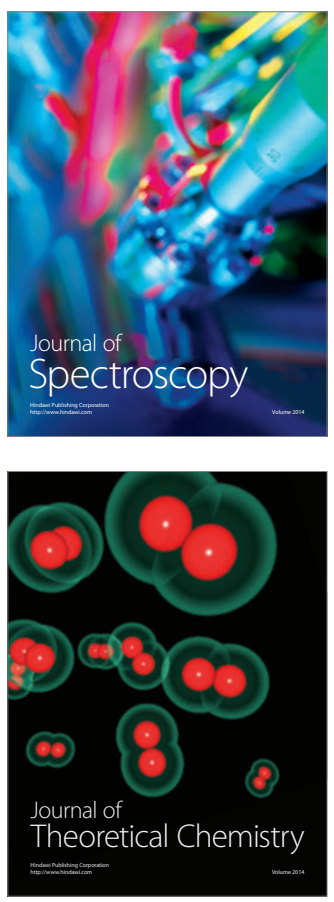
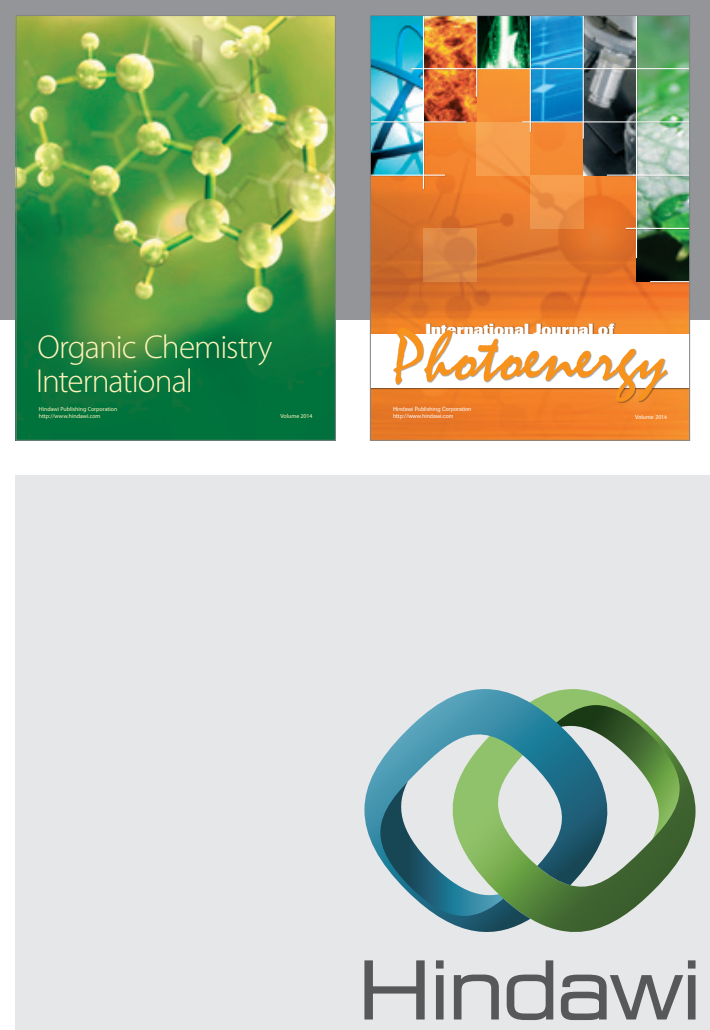

Submit your manuscripts at

http://www.hindawi.com
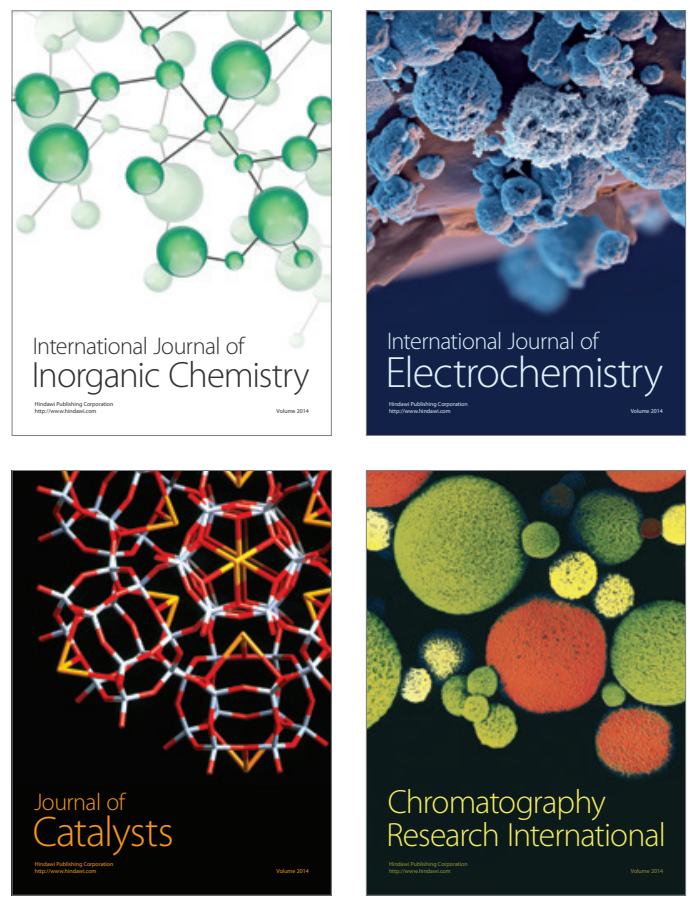
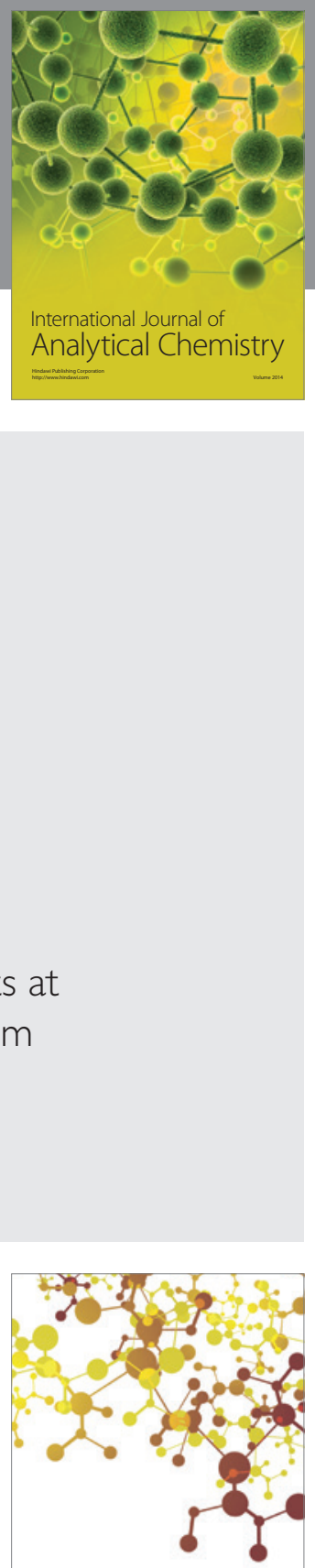

Journal of

Applied Chemistry
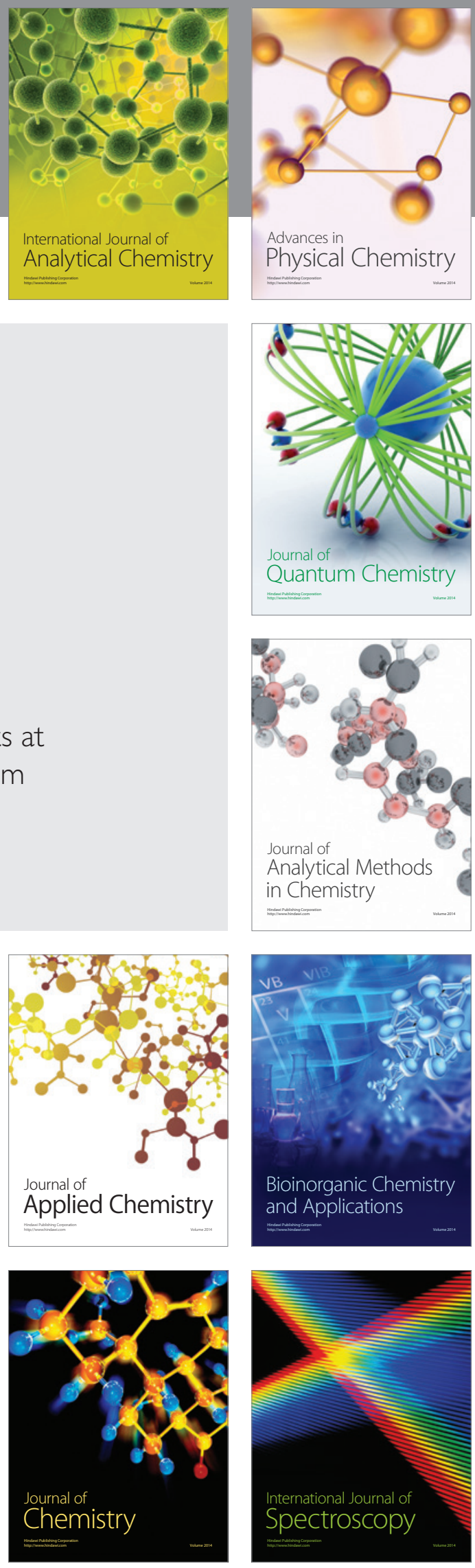